\title{
Supporting information for: Imaging and Steering Unidirectional Emission from Nanoantenna Array Metasurfaces
}

Klas Lindfors ${ }^{1,2,3}$, Daniel Dregely ${ }^{1,2}$, Markus Lippitz ${ }^{1,2,4}$, Nader Engheta $^{5}$, Michael Totzeck ${ }^{6}$, and Harald Giessen ${ }^{1}$

${ }^{1} 4$ th Physics Institute and Research Center SCoPE, University of Stuttgart, Pfaffenwaldring 57, 70550 Stuttgart, Germany

${ }^{2}$ Max Planck Institute for Solid State Research, Heisenbergstrasse 1, 70569 Stuttgart, Germany

${ }^{3}$ Department of Chemistry, University of Cologne, Luxemburger Strasse 116, 50939 Cologne, Germany

${ }^{4}$ Department of Physics, University of Bayreuth, Universitaetsstrasse 30, 95447 Bayreuth, Germany

${ }^{5}$ Department of Electrical and Systems Engineering, University of Pennsylvania, 200 South 33rd Street - ESE 203 Moore, Philadelphia, Pennsylvania 19104, USA

${ }^{6}$ Corporate Research and Technology, Carl Zeiss AG, Carl-Zeiss-Strasse 22, 73447 Oberkochen, Germany

Number of pages: 5

Number of figures: 1 


\section{Sample characterization}

Experiments were performed with a home-built fluorescence microscope. The experimental setup is shown in Fig. S1. We use $785 \mathrm{~nm}$ laser diodes (Spindler \& Hoyer, Germany, and Roithner Lasertechnik, Austria) as excitation sources. The single mode laser beam is expanded so that it completely fills the aperture of our objective. We use a Nikon CFI Plan Apo VC 100× oil immersion objective with a NA of 1.4 (Nikon, Japan) to focus onto the sample. From measurements on gold nanoparticles we determine the full width half maximum of the spot in the focus to 300 $\mathrm{nm}$. A polarizer and a removable waveplate control the incident polarization, and a mirror mounted on a galvanometer is used to adjust the position of incident beam with respect to the antennas.

Luminescence light is collected by the same objective and filtered from the excitation light with a dichroic beam-splitter (Semrock, USA) and long-pass filter (Chroma, USA). Wide-field images of the sample plane are taken with a nitrogen cooled CCD camera (Roper Scientific, USA). 


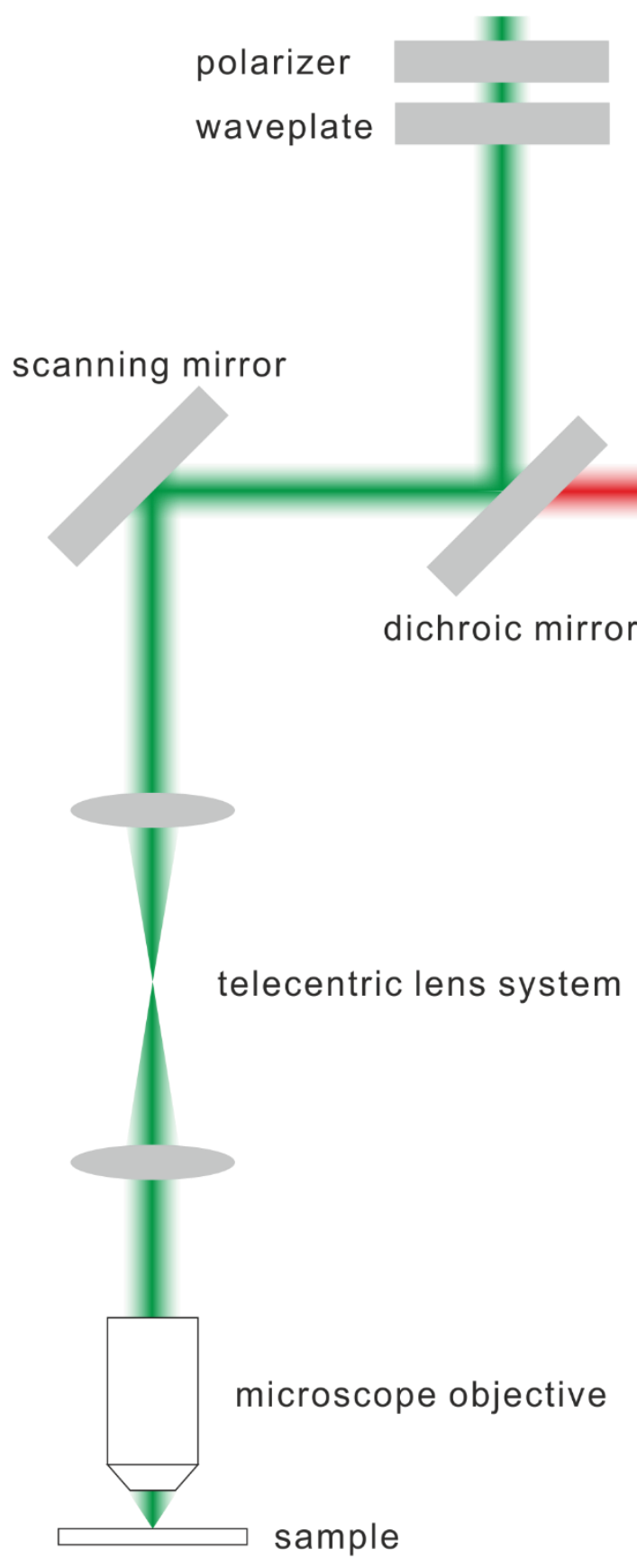

Figure S1. The experimental setup consists of a laser scanning microscope equipped with a CCD camera allowing recording wide-field images. Point-scanning images can be acquired using a single-photon counting module in order to find the structures to be measured. 


\section{Model for antenna emission pattern}

The incident field is calculated using the vectorial complex field description of a focused Gaussian laser beam ${ }^{\mathrm{S} 1}$. We describe the scattered field from our antenna array using the discrete-dipole approximation $^{\mathrm{S} 2}$, where each antenna element is considered as a single dipole. We take the polarizability of the dipoles from numerical simulations ${ }^{\mathrm{S} 3}$. The induced dipole moments of the antenna elements are calculated self-consistently, taking into account the coupling between the dipoles. The field radiated by the antenna array is the sum of the complex fields of all dipole elements. For the beamsteering, we take into account the shift of the incident driving field, which leads to a phase gradient across the.

The total intensity $I(\mathbf{r})$ around the transmitter is the squared absolute value of the sum of the complex incident field and the sum of the complex fields of all dipole elements

$$
I(\mathbf{r})=\left|\mathbf{E}_{\mathrm{inc}}(\mathbf{r})+a \sum_{k=1}^{N} \mathbf{E}_{\mathrm{dip}, k}(\mathbf{r})\right|^{2}
$$

where we introduce the parameter $a$ to weigh the relative strength of the incident field with respect to the field radiated by the antenna. In this way we take into account differences between the realized structures and the ideal antennas for which the polarizability was calculated numerically. The measured luminescence signal $s(t, \mathbf{r})$ does not scale linearly with the intensity $I(\mathbf{r})$ around the transmitter due to saturation and bleaching of the dye molecules. We take this into account using the nonlinear equation

$$
s\left(t_{0}, \mathbf{r}\right)=\int_{t_{0}}^{t_{0}+\Delta t} f(I(\mathbf{r}))\left[s_{1} e^{-c_{1} f(I(\mathbf{r}))\left(t-t_{0}\right)}+s_{2} e^{-c_{2} f(I(\mathbf{r}))\left(t-t_{0}\right)}\right] d t
$$

Here, $s\left(t_{0}, \mathbf{r}\right)$ is the signal obtained by collecting the emission for a duration $\Delta t$ starting at time $t_{0}$.

Saturation of the signal is taken into account in the term $f(I(\mathbf{r}))=\frac{I(\mathbf{r}) / I_{s}}{1+I(\mathbf{r}) / I_{s}}$, where $I_{s}$ is the saturation intensity. We assume a double exponential decay due to bleaching. The starting 
concentration of molecules bleaching with intensity dependent decay rates $c_{1} f(I(\mathbf{r}))$ and $c_{2} f(I(\mathbf{r}))$ are denoted $s_{1}$ and $s_{2}$, respectively.

\section{Fitting the antenna pattern}

We use Eq. (2) to fit the model to our experimental luminescence images. To obtain the parameters

characterizing the luminescent molecules $I_{s}, s_{1}, s_{2}, c_{1}$, and $c_{2}$, we fit the model to one dataset in each experiment. We then use these parameters to fit the model to the other datasets in the same experiment using $a$ as the only fitting parameter. This accounts for small variations in the fabricated devices.

\section{REFERENCES}

(S1) Novotny, L.; Hecht, B. Principles of Nano-Optics; Cambridge University Press: Cambridge, 2012.

(S2) Draine, B. T.; Flatau, P. J. Discrete-Dipole Approximation for Scattering Calculations. J. Opt. Soc. Am. A 1994, 11 (4), 1491.

(S3) CST Microwave Studios. http://www.cst.com 2012. 